\title{
Erratum
}

(Proc. Indian Acad. Sci. (Math. Sci.), Vol. 111, No. 4, November 2001, pp. 407-414)

\section{A variational proof for the existence of a conformal metric with preassigned negative Gaussian curvature for compact Riemann surfaces of genus $>1$}

\section{RUKMINI DEY}

Section 1 second paragraph should read: we minimize the functional

$$
S(\sigma)=\int_{M}(K(\sigma)-K)^{2} \mathrm{e}^{2 \sigma} \mathrm{d} \mu
$$

over $W^{2,2}(M) \ldots$. Using Sobolev embedding theorem we show that $S(\sigma)$ takes its absolute minimum on $W^{2,2}(M)$ which corresponds to a $C^{\infty}$ metric on $M$ of negative curvature $K$.

Section 2.1 should read: The functional $S(\sigma)=\int_{M}(K(\sigma)-K)^{2} \mathrm{e}^{2 \sigma} \mathrm{d} \mu$ is non-negative on $W^{2,2}(M)$, so that its infimum

$$
S_{0}=\inf \left\{S(\sigma), \sigma \in W^{2,2}(M)\right\}
$$

exists and is non-negative. Let $\left\{\sigma_{n}\right\}_{n=1}^{\infty} \subset W^{2,2}(M)$ be a corresponding minimizing sequence,

$$
\lim _{n \rightarrow \infty} S\left(\sigma_{n}\right)=S_{0}
$$

Our main result is the following

Theorem 0.1. Let $M$ be a compact Riemann surface of genus $g>1$. The infimum $S_{0}$ is attained at $\sigma \in C^{\infty}(M)$, i.e., the minimizing sequence $\left\{\sigma_{n}\right\}$ contains a subsequence that converges in $W^{2,2}(M)$ to $\sigma \in C^{\infty}(M)$ and $S(\sigma)=0$. The corresponding metric $\mathrm{e}^{\sigma} h \mathrm{~d} z \otimes \mathrm{d} \bar{z}$ is the unique metric on $M$ of negative curvature $K$.

Section 3, proof of the proposition (3.1) should read: ... Set $G(t)=S(\sigma+t \beta)-S_{0}$, where $\beta \in W^{2,2}(M) \ldots$. 